\title{
Viola-Jones based Detectors: How much affects the Training Set?
}

\author{
Modesto Castrillón-Santana, Daniel Hernández-Sosa, Javier Lorenzo-Navarro \\ SIANI \\ Edif. Central del Parque Científico Tecnológico \\ Universidad de Las Palmas de Gran Canaria \\ 35017 - Spain
}

\begin{abstract}
This paper presents a study on the facial feature detection performance achieved using the Viola-Jones framework. A set of classifiers using two different focuses to gather the training samples is created and tested on four different datasets covering a wide range of possibilities. The results achieved should serve researchers to choose the classifier that better fits their demands.
\end{abstract}

Keywords: Viola-Jones detectors, facial feature detection, training sets

\section{Introduction}

The Viola-Jones face detector [16] has been extensively used thanks to the implementation available [10] in the OpenCV (Open Computer Vision) library [7]. However, Viola and Jones designed a general object detection framework that can be used for other objects. Its OpenCV implementation allows researchers to train their own classifier(s). Previously, during the sample gathering stage a large set of images is built with samples containing the object to detect (positive samples) and others not containing the target (negative samples).

Positive and negative samples gathering, data annotation, data preparation and training are uncomfortable and slow tasks that have been summarized in different brief tutorials, e.g. [14]. In this sense more recent implementations [2] have tried to keep the performance while reducing the training and test processing.

Within the facial analysis scenario, facial feature detection is a topic of interest as is may serve to reduce false positive detections when using a face detector, or to better align a detected face. Thanks to OpenCV, different face related classifiers are available to a large community of researchers $[7,11]$. Their performance have already been compared with different test sets, but no details related to the samples used during their training stage are available.

In this paper we train different facial feature classifiers making use of training sets of different nature, and test them with a large heterogeneous collection of face datasets, in terms of pose, illumination and resolution. We aim at providing researchers hints about how to build a detector for their particular application characteristics. 
Section 2 summarizes the Viola-Jones object detection framework. The different datasets are briefly described in Section 3 and the results and conclusions in sections 4 and 5 respectively.

\section{Viola-Jones general object detection framework}

Automatic face detectors have received researchers attention in last years, evolving notoriously $[5,17]$. In this sense recent approaches $[13,16]$ have reduced dramatically the processing latency at high levels of accuracy, without requiring restricted heuristics based on cues such as skin color or motion. These approaches make use of a sliding window that is shifted at different scales across the whole image. Each time the area is checked with a classifier to verify whether the target pattern is present.

Following the sliding window approach, face detectors based on the framework described in [16] have achieved remarkable results while becoming well known thanks to the implementation [10] integrated in OpenCV [7]. This framework is based on the idea of a boosted cascade of weak classifiers, i.e. each one has a high detection ratio, with a reduced true reject ratio. Each classifier uses a set of Haar-like features, acting as a filter chain. Only those image regions that manage to pass through all the stages of the detector are considered as containing the target. For each stage in the cascade, a separate subclassifier is trained to detect almost all target objects while rejecting a certain fraction of those non-object patterns that have been incorrectly accepted by previous stage classifiers.

Theoretically for a cascade of $K$ independent classifiers, the resulting detection rate, $D$, and the false positive rate, $F$, of the cascade are given by the combination of each single stage classifier rates:

$$
D=\prod_{i=1}^{K} d_{i} \quad F=\prod_{i=1}^{K} f_{i}
$$

Each stage classifier is selected considering a combination of features which are computed on the integral image. These features are reminiscent of Haar wavelets and early features of the human visual pathway such as center-surround and directional responses. The implementation [10] integrated in the OpenCV [7] extends the original feature set [16].

With this approach, given a 20 stage detector designed for refusing at each stage $50 \%$ of the non-object patterns (target false positive rate) while falsely eliminating only $0.1 \%$ of the object patterns (target detection rate), its expected overall detection rate is $0.999^{20} \approx 0.98$ with a false positive rate of $0.5^{20} \approx$ $0.9 * 10^{-6}$. This schema allows a high image processing rate, due to the fact that background regions of the image are quickly discarded, while spending more time on promising object-like regions. Thus, the detector designer chooses the desired number of stages, the target false positive rate and the target detection rate per stage, achieving a trade-off between accuracy and speed for the resulting classifier. 
Given an input image, the resulting classifier will report the presence and location of the object of interest.

The availability of different tutorials, e.g. [14], help OpenCV users to collect, annotate and structure the data before building the different classifiers that are later tested with an independent set of images.

\section{Datasets}

Being interested in testing a facial feature detector performance, we previously selected some face datasets to test. Different datasets have been used in the past to analyze face detection performance. However, we wanted to cover a wide range of situations to better characterize the classifiers under study. For that purpose four datasets of facial images have been selected:

- The CMU database [13] contains a collection of heterogeneous images divided into four different subsets test, new-test, low-res and rotated combining the test sets of Sung and Poggio [15] and Rowley, Baluja and Kanade [12]. The dataset and the annotation data corresponding to 721 faces can be obtained at [3].

- More recently initiatives such as FIW [6] have introduced new challenging situations to test the performance of the face related detectors with much larger datasets. The availability of annotation data [8] increases the number of annotated faces in real situations. In this dataset the authors provide face location information in terms of ellipses.

- The Yale Face database [1] contains a homogeneous collection of face images in different illumination conditions.

- Facity ${ }^{1}$ is an online photo project presenting high quality frontal face images with natural illumination, no facial expression and open eyes.

Table 1 summarizes the number of images and faces available in each dataset. CMU and FDDB datasets can contain more than one face per image. The average image size (it is fixed for Yale and Facity sets), the average eye distance (in pixels) of each face annotated and the dataset standard deviation is also provided to indicate the dataset variability.

Excepting the CMU dataset, no other dataset is provided with information related to the facial features, therefore we have roughly annotated the center point of the main facial features: eyes, nose and mouth.

The criterion adopted to consider a facial feature, $f_{i}$, detection as correct, is that the euclidean distance between the annotated location, $\operatorname{pos}_{f_{i} \text {, annotated }}$, and the detected location, $\operatorname{pos}_{f_{i} \text {,detected }}$, must be lower than one fourth the actual eye distance. This criterion was used to estimate the eye detection success originally in [9].

\footnotetext{
$\overline{{ }^{1} \text { www.facity.com }}$
} 


\begin{tabular}{|llllll|}
\hline Dataset Number of Average image Number of & \multicolumn{2}{c|}{ Average eye Standard } \\
& images & dimension & annotated faces distance & deviation \\
\hline FDDB & 2845 & $377 \times 399$ & 5171 & 99 & 177 \\
CMU & 180 & $421 \times 422$ & 721 & 64 & 203 \\
Yale & 165 & $320 \times 243$ & 165 & 55 & 3.4 \\
Facity & 3114 & $600 \times 600$ & 3114 & 206 & 14 \\
\hline
\end{tabular}

Table 1. Datasets statistics. The average image dimension, average eye distance and standard deviation are expressed in pixels.

\section{Experiments}

\subsection{Classifiers}

For each facial feature (eyes, nose and mouth) we have made use of two different training datasets:

- Set A: A collection of 6000 heterogeneous images taken randomly from the web. Using this dataset four different classifiers were trained: left eye, right eye, nose and mouth. These classifiers are already included in the current OpenCV release and have been analyzed in [4].

- Set B: A subset of 2300 faces of the Facity collection. Using this dataset different classifiers were trained: left eye, right eye, iris, nose, mouth, left mouth corner and right mouth corner.

For both training sets the flipped image was also used for training purposes, therefore we had around 12000 positive samples for the first family and around 4600 for the second. For both configurations around 15000 images were used as negative samples.

\subsection{Results}

The receiver operating characteristic (ROC) curve of each classifier is computed applying first the original release of each classifier, and two variants reducing its number of stages. Theoretically, this action must increase both correct, $D$, and false, $F$, detection rates.

The processing cost and detection precision are reflected in Table 2 for each classifier. The processing cost indicates the total time needed, in seconds, to process the whole dataset in a Core $2 \mathrm{PC}$. The precision is related to the actual eye distance of the face, only for those detections considered true detections. It is observed that the classifiers computed making use of the training set B are much faster. This is justified by the simplicity of the resulting classifiers on each stage, the simpler the training images, the faster the resulting classifier. These classifiers are also similar or slightly more precise than those obtained using the training set A. They are particularly much more precise for images of similar nature than those used to train the classifiers, i.e. Facity. 


\begin{tabular}{|ccccccccc|}
\hline Classifier & \multicolumn{3}{c}{ FDDB } & \multicolumn{3}{c|}{ CMU } & \multicolumn{3}{c|}{ Yale } & \multicolumn{3}{c|}{ Facity } \\
& \multicolumn{8}{c}{ Time Precision Time Precision Time Precision Time Precision } \\
\hline \hline Right eye & 550 & 0.073 & 46 & 0.054 & 14 & 0.03 & 1546 & 0.02 \\
Left eye & 563 & 0.072 & 47 & 0.068 & 15 & 0.04 & 1625 & 0.04 \\
Nose & 927 & 0.069 & 72 & 0.11 & 19 & 0.04 & 1990 & 0.05 \\
Mouth & 677 & 0.17 & 58 & 0.11 & 16 & 0.06 & 1672 & 0.13 \\
\hline \multicolumn{8}{c}{ Classifiers trained with set B } \\
Iris & 83 & 0.04 & 8 & - & 2 & 0.04 & 229 & 0.009 \\
Left Eye & 63 & 0.01 & 7 & - & 1.3 & - & 185 & 0.01 \\
Right eye & 61 & 0.09 & 7 & - & 1.4 & - & 174 & 0.006 \\
Nose & 90 & 0.1 & 9 & 0.16 & 2 & 0.07 & 233 & 0.08 \\
Left mouth & 88 & - & 9 & 0.05 & 2 & 0.04 & 256 & 0.02 \\
Right mouth & 100 & - & 10 & 0.04 & 2 & 0.04 & 289 & 0.02 \\
Mouth & 86 & - & 9 & 0.08 & 2 & 0.09 & 231 & 0.02 \\
\hline
\end{tabular}

Table 2. Classifier processing cost in seconds per dataset and positive detection precision, relative to the actual eye distance.

Those classifiers trained using set B present two important characteristics, they are faster, almost ten times for some facial features, and similar or more precise. Unfortunately, they are not so reliable to the whole dataset collection as seen in Figures 1-5. Their respective areas under the ROC curve are smaller than those presented bu the family of classifies computed with set A. To analyze each feature, Figure 1 and 2 compares the detection rate of the two classifiers specialized in the eye detection. The detectors based on the set A perform similarly for both eyes. However they are worst, as expected, for those datasets with unrestricted pose, while being really reliable with the frontal face datasets: Facity and Yale. On the other side, the left eye detector based on the set B offers a poor performance even for the Facity dataset. The iris detector presents better performance, and a reduced false detection rate, but far from that achieved using set $\mathrm{A}$.

The nose detection rates are presented in Figure 3. The behavior for the set A is similar to that observed for the eye pattern. The classifiers obtained with set $\mathrm{B}$ are now behaving better for the Yale and Facity but they never reach the reliability exhibited by those trained with set A. However, the reader must remember that this detector is much faster.

A similar performance is observed for the mouth detection, see Figure 4. We have also included the performance of the mouth corner classifiers, see Figure 5. The latter is only sensitive for the Facity dataset.

\section{Conclusions}

We have trained facial features detectors using two different kind of samples to build the training set. The training set A contains heterogeneous images under uncontrolled conditions, in contrast with the homogeneous training set B. 

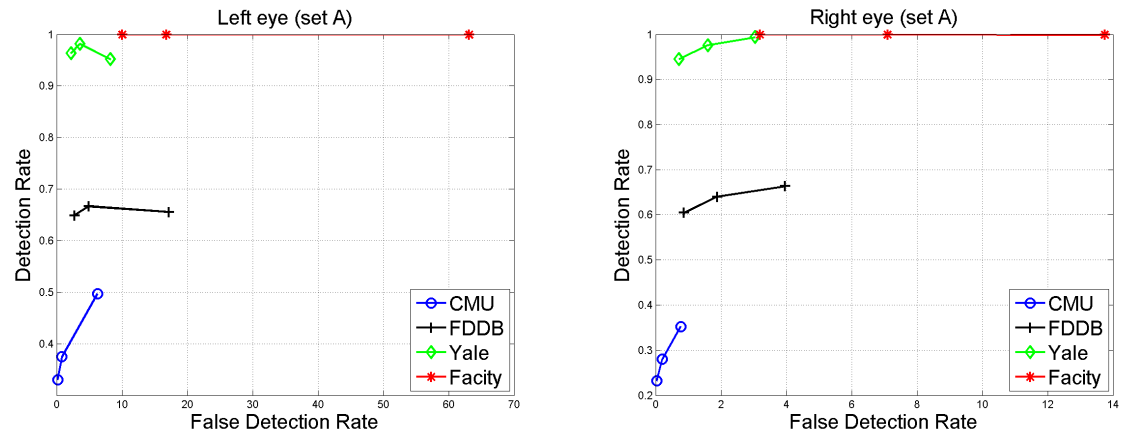

Fig. 1. Performance achieved using the left and right eye detector computed with the training set A.
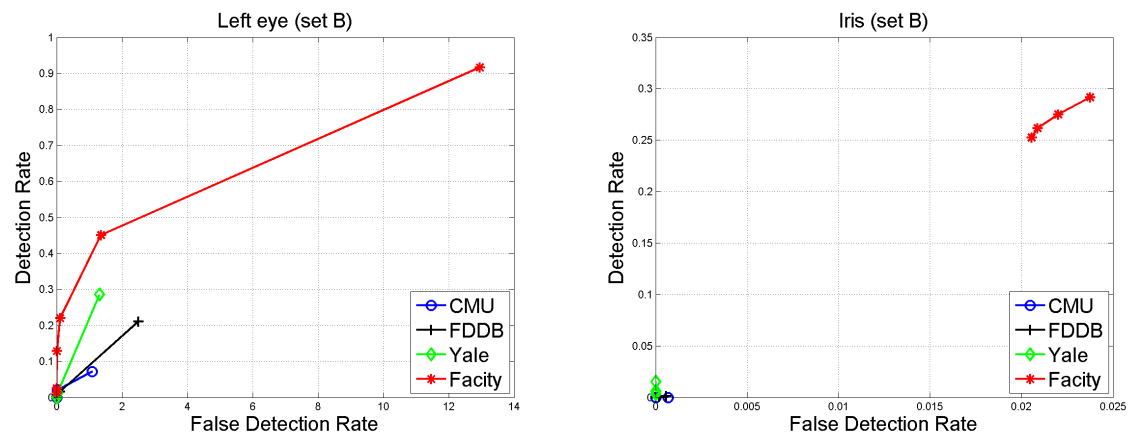

Fig. 2. Left) Left eye detection performance using the training set B. Right) Performance achieved using the iris detector computed with the training set B.

The results achieved with the training set $\mathrm{A}$ are more reliable than those achieved with the training set B. Those classifiers trained with set B are faster (almost ten times), with similar or better precision and present lower false detection rates, but their ROC curves suggest a clearly worse performance. They exhibit close performance only for datasets containing images of similar nature to those used for training. We can conclude that the training set does not encloses enough appearance information to build a robust facial feature detector

For future work we plan to combine the detectors and even the training sets. The effort must be done in terms of speeding up the process while keeping similar performance to those achieved with the training set A.

\section{Acknowledgments}

This work was partially supported by the Spanish Ministry of Science and Innovation funds (TIN2008-06068). 

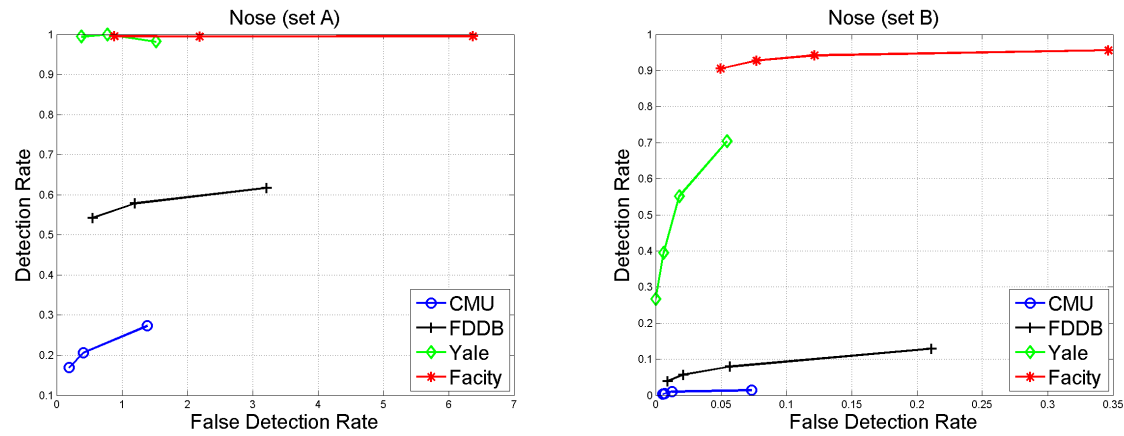

Fig. 3. Left) Nose detection performance using the training set A. Right) Nose detection performance using the training set $\mathrm{B}$.
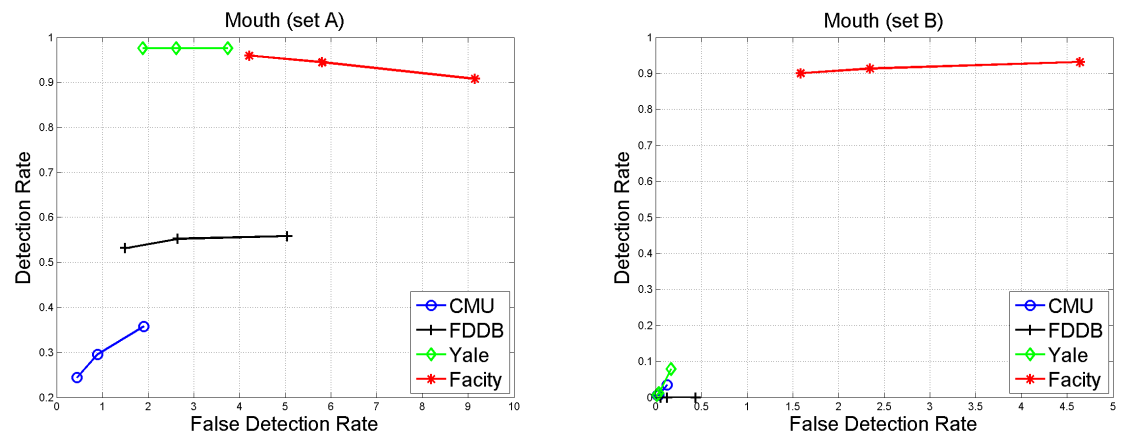

Fig. 4. Left) Mouth detection performance using the training set A. Right) Mouth detection performance using the training set B.

\section{References}

1. Belhumeur, P., Hespanha, J., Kriegman, D.: Eigenfaces vs. Fisherfaces: Recognition using class specific linear projection. IEEE Trans. on PAMI 19(7), 711-720 (1997)

2. Brubaker, S.C., Wu, J., Sun, J., Mullin, M.D., Rehg, J.M.: On the design of cascades of boosted ensembles for face detection. International Journal of Computer Vision 77, 65-86 (2008)

3. Carnegie Mellon University: CMU/VACS image database: Frontal face images. http://vasc.ri.cmu.edu/idb/html/face/frontal_images/ index.html (1999), last accesed 5/11/2007

4. Castrillón, M., Déniz, O., Hernández, D., Lorenzo, J.: A comparison of face and facial feature detectors based on the violajones general object detection framework. Machine Vision and Applications (in press) (2010)

5. Hjelmas, E., Low, B.K.: Face detection: A survey. Computer Vision and Image Understanding 83(3), 236-274 (2001), http://dx.doi.org/10.1006/cviu. 2001. 0921 

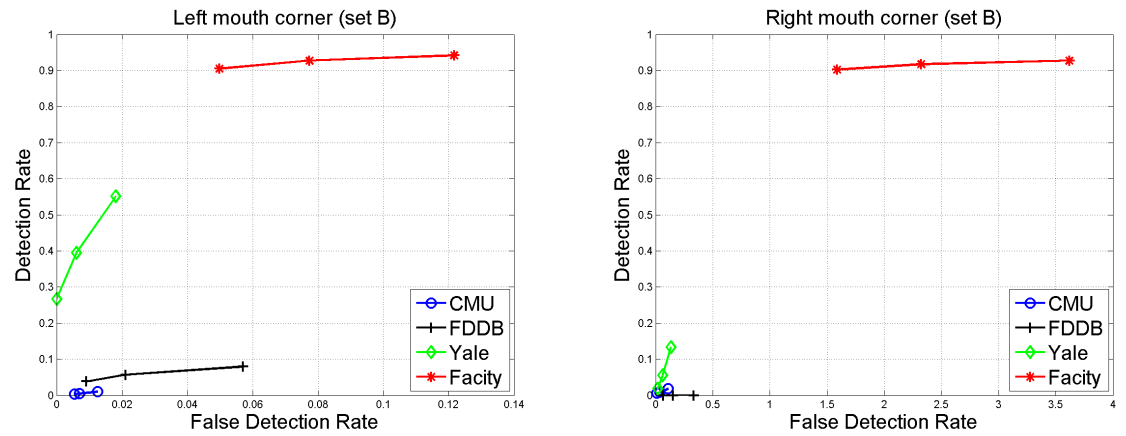

Fig. 5. Mouth corners detection performance using the training set B.

6. Huang, G.B., Ramesh, M., Berg, T., Learned-Miller, E.: Labeled faces in the wild: A database for studying face recognition in unconstrained environments. Tech. Rep. 07-49, University of Massachusetts, Amherst (October 2007)

7. Intel: Intel Open Source Computer Vision Library, v2.1. http://sourceforge.net/projects/opencvlibrary/ (April 2010), (last visited June 2010)

8. Jain, V., Learned-Miller., E.: Fddb: A benchmark for face detection in unconstrained settings. Tech. rep., University of Massachusetts, Amherst. (2010)

9. Jesorsky, O., Kirchberg, K.J., Frischholz, R.W.: Robust face detection using the Hausdorff distance. Lecture Notes in Computer Science. Procs. of the Third International Conference on Audio- and Video-Based Person Authentication 2091, 90-95 (2001)

10. Lienhart, R., Maydt, J.: An extended set of Haar-like features for rapid object detection. In: IEEE ICIP 2002. vol. 1, pp. 900-903 (September 2002)

11. Reimondo, A.: Haar cascades repository. http://alereimondo.noip.org/OpenCV/34 (2007), (last visited April 2010)

12. Rowley, H.A., Baluja, S., Kanade, T.: Neural network-based face detection. IEEE Trans. on Pattern Analysis and Machine Intelligence 20(1), 23-38 (1998)

13. Schneiderman, H., Kanade, T.: A statistical method for 3d object detection applied to faces and cars. In: IEEE Conference on Computer Vision and Pattern Recognition. pp. 1746-1759 (2000)

14. Seo, N.: Tutorial: OpenCV haartraining (rapid object detection with a cascade of boosted classifiers based on haar-like features). http://note.sonots.com/ SciSoftware/haartraining.html, (last visited June 2010)

15. Sung, K.K., Poggio, T.: Example-based learning for view-based human face detection. IEEE Trans. on Pattern Analysis and Machine Intelligence 20(1), 39-51 (January 1998)

16. Viola, P., Jones, M.J.: Robust real-time face detection. International Journal of Computer Vision 57(2), 151-173 (May 2004)

17. Yang, M.H., Kriegman, D., Ahuja, N.: Detecting faces in images: A survey. Transactions on Pattern Analysis and Machine Intelligence 24(1), 34-58 (2002), http://dx.doi.org/10.1109/34.982883 